\title{
Synthesis of well-defined conjugated oligomers for molecular electronics
}

\author{
LeRoy Jones II, Darren L. Pearson, Jeffry S. Schumm, and James M. Tour* \\ Department of Chemistry and Biochemistry, University of South Carolina \\ Columbia, South Carolina 29208
}

\begin{abstract}
Described are methods for the rapid syntheses of conjugated oligomers of precise length and constitution by an iterative divergent/convergent approach. The syntheses can be carried out in solution or on a polymer support. The properties of the conjugated oligomers are presented as well there potential to act as molecular wires.
\end{abstract}

Molecular electronics-based computation has attracted interest because the ultimate computational system would consist of logic devices that are ultra dense, ultra fast, and molecular-sized. Even though state-of-the-art nanopatterning techniques allow lithographic probe assemblies to be engineered down to the $100 \AA$ gap regime, the issue of electronic conduction based upon single or small packets of molecules has not been addressed and the feasibility of molecular electronics remains theoretically controversial $(1,2)$. In an attempt to assess the possibility of molecular wire conduction by spanning the $100 \AA$ probe gaps with small packets of molecules, we recently synthesized the oligo( $\alpha$-thiophene ethynylene)s by the rapid iterative divergent/convergent approach using, successively, the same three sets of reaction conditions (Fig. 1) (3,4). The organometallic $\mathrm{Pd} / \mathrm{Cu}$ coupling methods have proved to be most valuable for the needed coupling reactions. The monomer through 16-mer have been characterized spectroscopically. While the tetramer and octamer afforded molecular ions by direct exposure via electron impact mass spectrometry, neither this method nor fast atom bombardment or electrospray mass spectrometry sufficed for obtaining a molecular ion of the final 16-mer. However, MALDI-MS did afford an $M+1$ peak and this information proved to be most important for the definitive characterization $(3,5)$.

We used a similar method to prepare oligo(p-phenylene ethynylene)s (Fig. 2) (6). The linear oligo(phenylene ethynylene) arrangement should minimize undesired conformational movement during adhesion and testing between nanofabricated probes. In order to further streamline the synthesis, we have utilized the iterative divergent/convergent approach on a polymer support (7). In this manner, the purification steps could be significantly reduced.

The oligo(arylene ethynylene)s can be functionalized at the ends to serve as "molecular alligator clips" that are required for surface contacts to metal probes for molecular electronics studies. For example, we have functionalized the ends of the octa( $\alpha$-thiophene ethynylene) with thiol moieties, shown in protected form (Fig. 3), for adhesion to gold probes by self-assembly methods. We have also synthesized isonitrile end groups for adhesion to tungsten probes (8). Thus, the iterative divergent/convergent approach permitted both the facile construction of the potential molecular wires as well as providing compounds with ends that could be readily functionalized.

Since the potential molecular wires must bridge two gold electrodes, the question as to how these rigid rod difuctional oligomers will order on gold surfaces comes into question. For example, will the oligomers bridge the gold-gold slectrode gaps, as in Fig. 4a, or will they remain nearly parallel to the surface of the gold by either dithiol or aromatic adsorption to the gold surfaces, as in Figs. $4 \mathrm{~b}$ and $4 \mathrm{c}$, respectively? By making self-assembled monolayers on gold surfaces, we have demonstrated using

ellipsometry, XPS, and grazing angle IR measurements, that the rigid rod systems predominantly stand perpendicular to the surface; the thiol groups dominating the adsorption sites on the gold. Even when the oligomers were $\alpha, \omega$-dithiol-substituted, the rigid molecules tended to stand on end as judged by the ellipsometric thickness of the adsorbate layer (9). Therefore, using these precisely length conjugated oligomers, we are well on our way to addressing the potential of molecular electronics. 

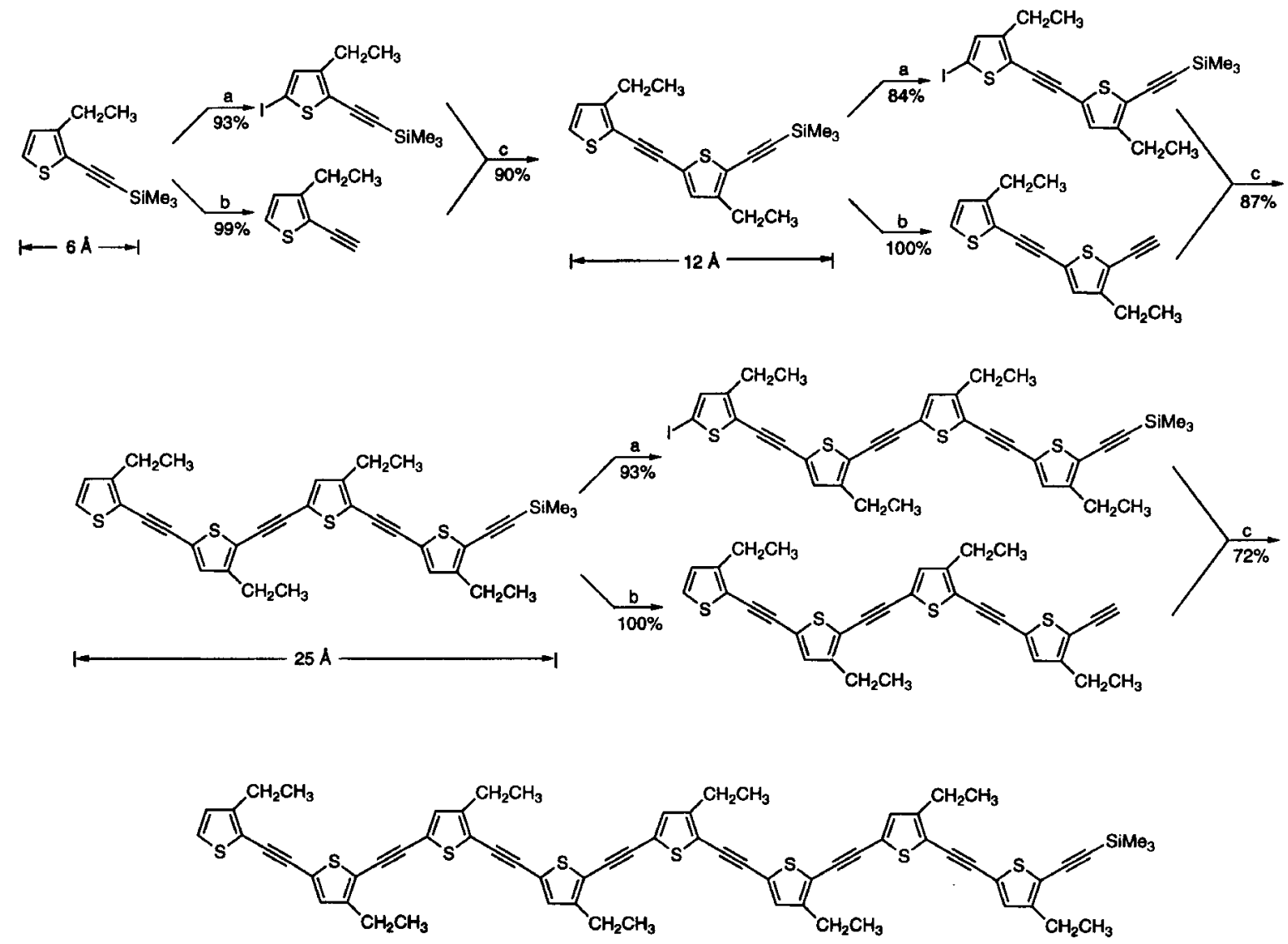

$50 \mathrm{~A}$
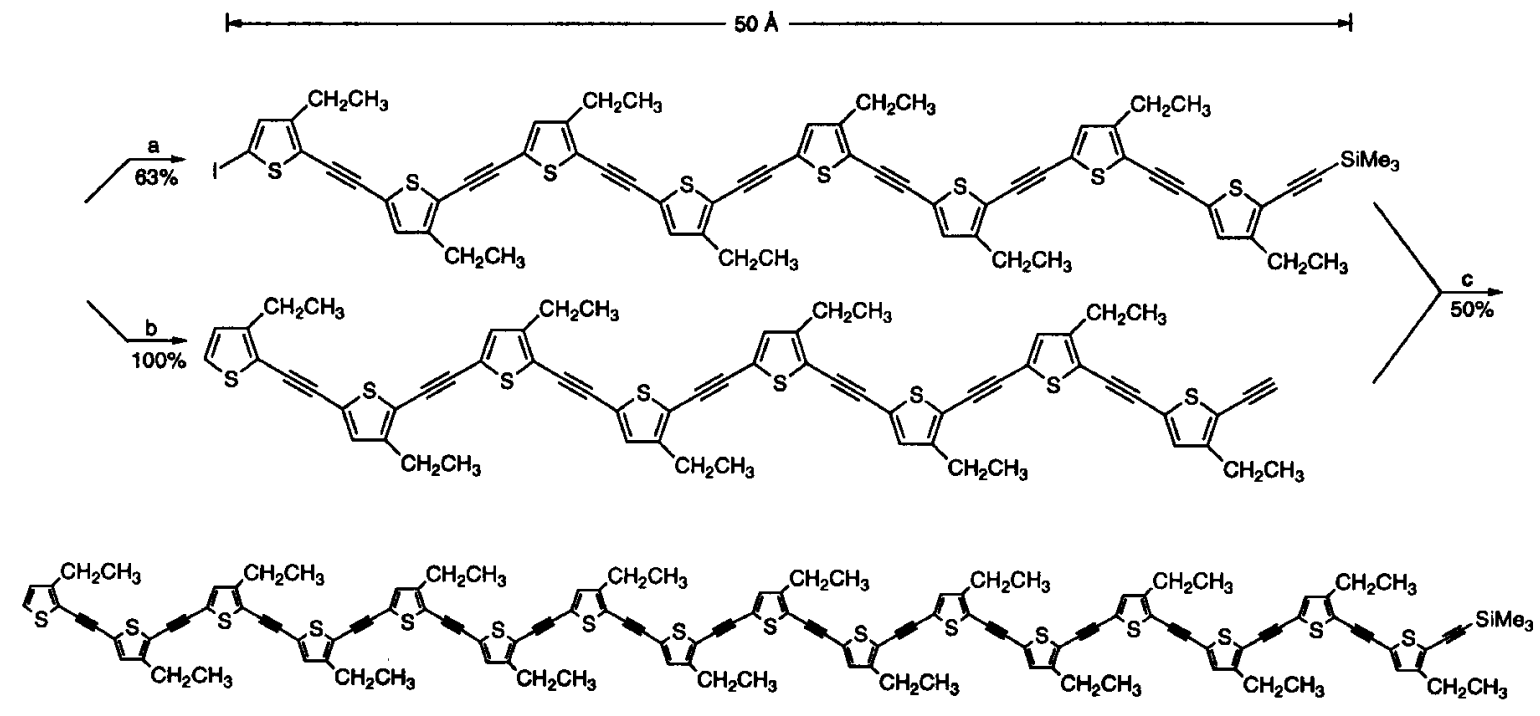

Reagents: (a) LDA, $\mathrm{Et}_{2} \mathrm{O},-78^{\circ}$ to $0^{\circ} \mathrm{C} ; \mathrm{l}_{2},-78^{\circ}$. (b) $\mathrm{K}_{2} \mathrm{CO}_{3}, \mathrm{MeOH}, 23^{\circ}$. (c) $\left.\mathrm{Cl}_{2} \mathrm{Pd}_{(\mathrm{PPh}}\right)_{2}(2 \mathrm{~mol} \%), \mathrm{Cul}(1.5 \mathrm{~mol} \%), \mathrm{THF}, j \mathrm{Pr}_{2} \mathrm{NH}$.

Figure 1. Iterative divergent/convergent synthesis of oligo(thiophene ethynylene)s. 

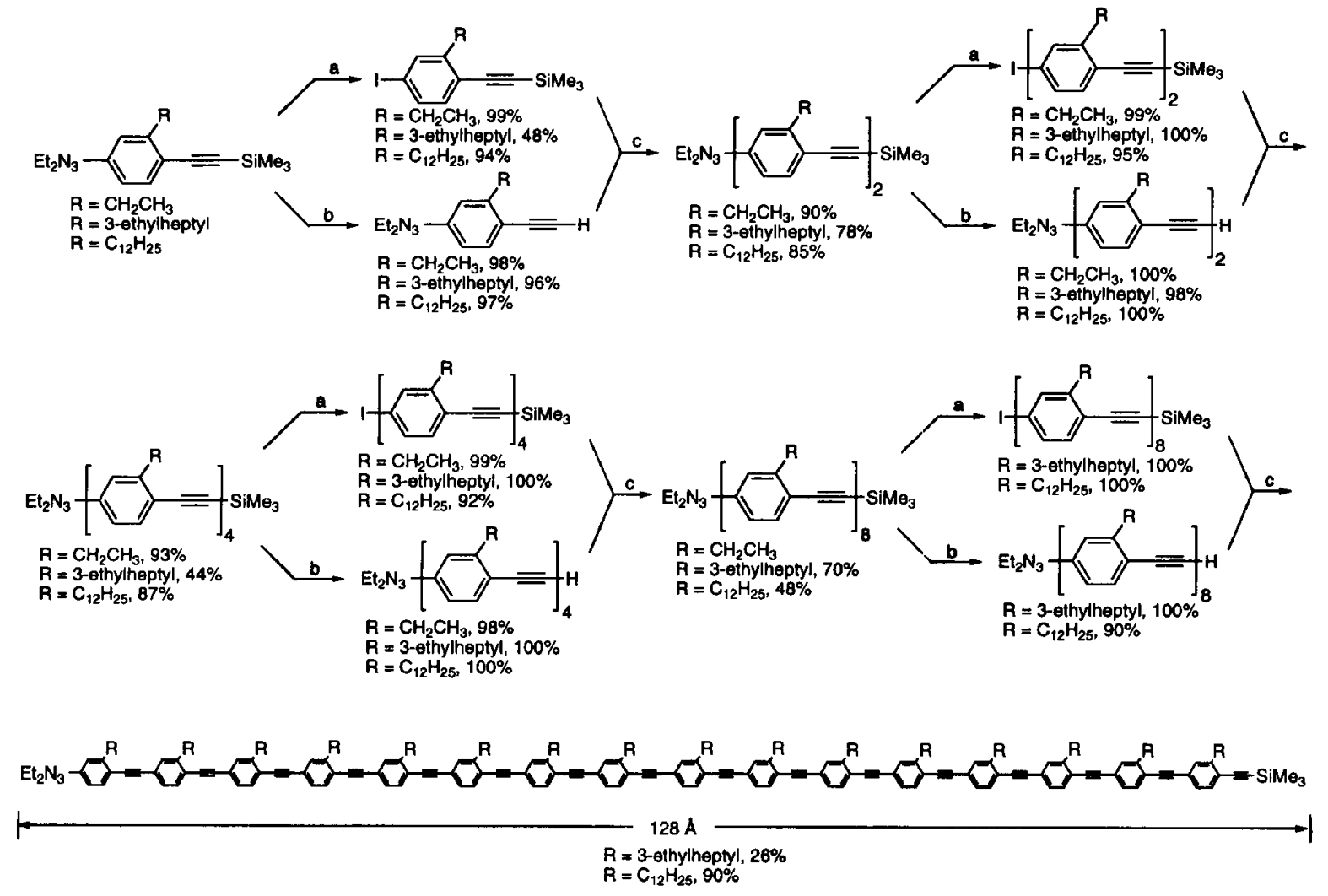

Reagents: a. Mel as solvent, $120^{\circ} \mathrm{C}$ in a screw cap tube. b. $\mathrm{K}_{2} \mathrm{CO}_{3}, \mathrm{MeOH}, 23^{\circ} \mathrm{C}$ or $n-\mathrm{Bu}_{4} \mathrm{NF}, \mathrm{THF}, 23^{\circ} \mathrm{C} \mathrm{c} . \mathrm{Pd}(\mathrm{dba})_{2}(5 \mathrm{~mol} \%), \mathrm{Cul}$ $(10 \mathrm{~mol} \%), \mathrm{PPh}_{3}(20 \mathrm{~mol} \%), i \mathrm{Pr}_{2} \mathrm{NH} / \mathrm{THF}(1: 5), 23^{\circ} \mathrm{C}$.

Figure 2. Iterative divergent/convergent synthesis of oligo(phenylene ethynylene)s.
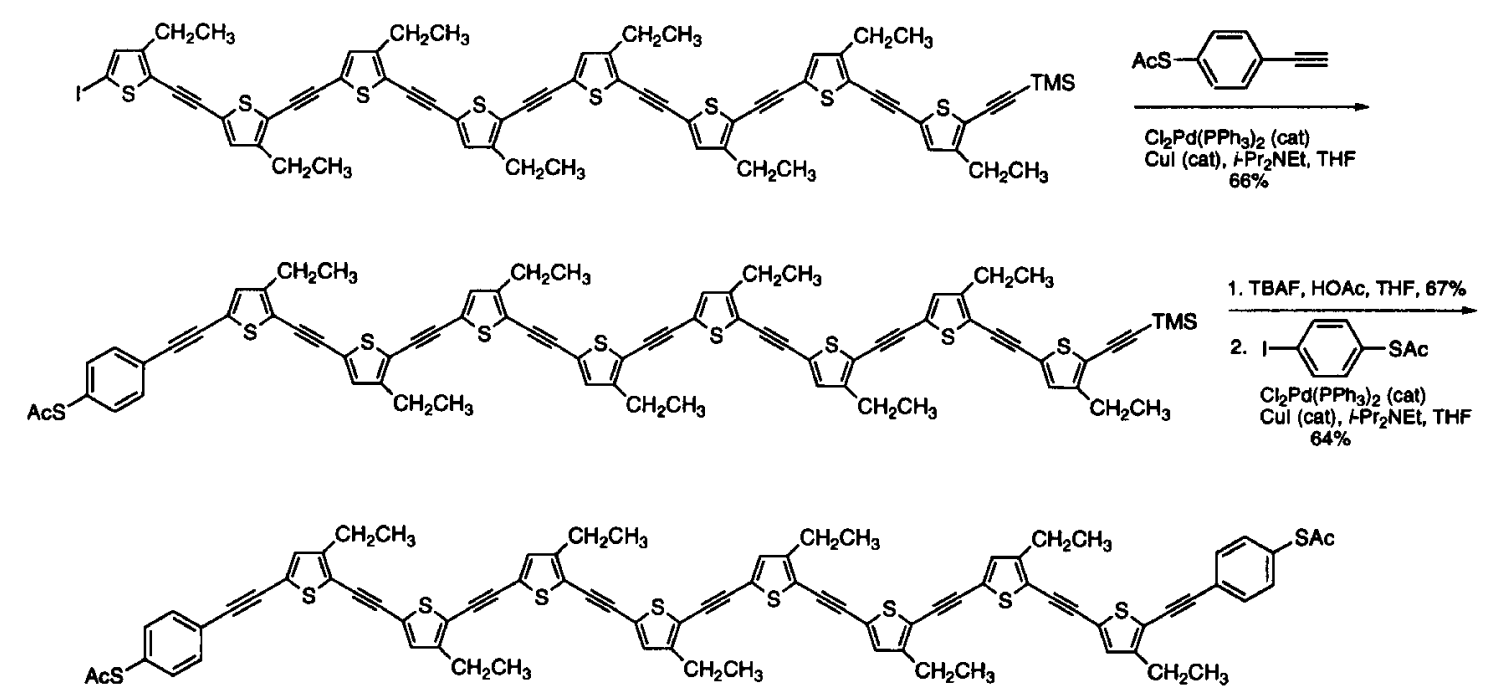

Figure 3. Attachment of "molecular alligator clips" to potential molecular wires. 
(a)

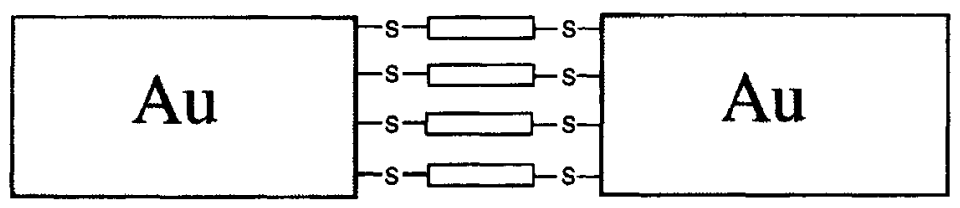

(b)
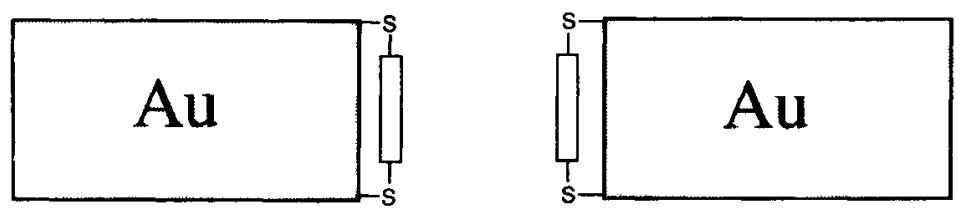

(c)
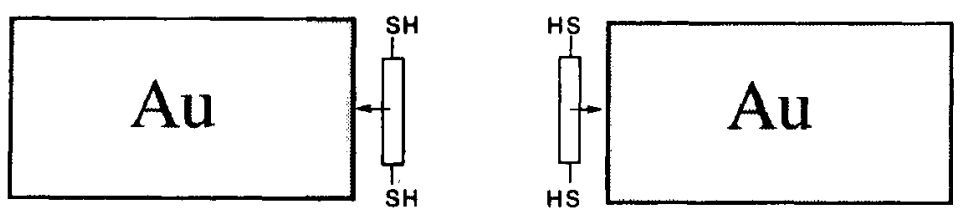

Figure 4. Possible modes of adsorption between dithiol-terminated rigid rod oligomers and proximate gold probes.

In addition to these conjugated molecules serving as potential molecular wires, their optical and chromatographic properties proved to be most intriguing. An optical saturation or near saturation of the systems occurred by the octamer stage so that doubling the conjugation length to the 16-mer caused little or no change in the position of the absorbance maximum $(3,6)$. The results of the size exclusion chromatography (SEC) were compared with the actual molecular weights of the oligomers. SEC is not a direct measure of MW, but a measure of the hydrodynamic volume. Thus, by SEC using randomly coiled polystyrene standards, the number average molecular weights $\left(M_{n}\right)$ of rigid rod polymers are usually greatly inflated relative the actual molecular weights. Accordingly, the SEC recorded $M_{\mathrm{n}}$ values of the octamers and 16-mers were much greater than the actual molecular weights. Also, as we would predict, the monomer, dimer, and tetramer had $M_{\mathrm{n}}$ values that were reasonably close to the actual molecular weights because they are in the low MW region, prior to significant polystyrene coiling. In all cases, the SEC-determined values of $M_{\mathrm{W}} / M_{\mathrm{n}}=1.02-1.07$. Thus this plot can serve as a useful calibration chart for very rigid linear oligomers as they compare to polystyrene standards by $\operatorname{SEC}(3,6)$.

In summary, organometallic methods have provided a facile entry into the nano-architectures needed for molecular electronics research.

Acknowledgment:

This work was funded by the Advanced Research Projects Agency.

References:

1. M. A. Reed and W. P. Kirk, Eds. Nanostructure Physics and Fabrication; Academic Press: San Diego, (1989).

2. W. P. Kirk and M. A. Reed, Eds. Nanostructures and Mesoscopic Systems; Academic: San Diego, (1992).

3. D. L. Pearson, J. S. Schumm, and J. M. Tour. Macromolecules 27, 2348 (1994).

4. G. Wegner, In Thermoplastic Elastomers, A Comprehensive Review, N. R. Legge, G. Holden and H. E. Schroeder, Eds.; Hanser: New York, 405 (1987).

5. H. S. Creel. Trends in Polym. Sci. 1, 336 (1993).

6. J. S. Schumm, D. L. Pearson and J. M. Tour. Angew. Chem., Int. Ed. Engl. 33, 1360 (1994).

7. J. K. Young, J. C. Nelson and J. S. Moore. J. Am. Chem. Soc. 116, 10841 (1994).

8. J. S. Schumm, L. Jones II, D. L. Pearson, R. Hara and J. M. Tour. Polym. Prepr. (Am. Chem. Soc., Div. Polym. Chem.) 35(2), 687 (1994).

9. J. M. Tour, L. Jones II, D. L. Pearson, J. S. Lamba, T. P. Burgin, G. W. Whitesides, D. L. Allara, A. N. Parikh and S. Atre. J. Am. Chem. Soc., in press. 